\title{
The Frequency-Dependence of Pre- and Postganglionic Nerve Stimulation of Pig and Rat Bladder
}

\author{
Darryl Kitney ${ }^{1}$, John Crook $^{1}$, Basu Chakrabarty ${ }^{1}$, Anthony Kanai ${ }^{2}$, Bahareh Vahabi ${ }^{3}$, Thelma Lovick ${ }^{1}$, Christopher Fry \\ ${ }^{1}$ School of Physiology, Pharmacology and Neuroscience, University of Bristol, Bristol, UK \\ ${ }^{2}$ School of Medicine, University of Pittsburgh, Pittsburgh, PA, USA \\ ${ }^{3}$ Department of Applied Sciences, University of the West of England, Bristol, UK
}

Purpose: The urinary bladder generates phasic contractions via action potentials generated in pre- and then postganglionic neurons. Whilst the frequency-dependence of postganglionic neurons to generate contractions has been quantified, the dynamic range of preganglionic neurons is less clear and if intramural ganglia exert frequency-dependent modulation of transmission between pre- and postganglionic neurons. The phosphodiesterase type 5 inhibitor sildenafil reduces neurotransmitter release from postganglionic fibres to detrusor smooth muscle and an additional question was if there was also a preganglionic action. This study aimed to compare the frequency range of bladder contractile activation by pre- and postganglionic stimulation in pig and rat bladders and if sildenafil exerted additional preganglionic actions.

Methods: An arterially-perfused ex vivo pig bladder preparation was used for preganglionic (pelvic nerve) and mixed preand postganglionic (direct bladder wall) stimulation at $36^{\circ} \mathrm{C}$ and postganglionic mediated contractions achieved by field-stimulation of in vitro isolated detrusor strips. With rats, pelvic nerve stimulation was carried out in vivo and postganglionic stimulation also with isolated detrusor strips.

Results: All contractions were abolished by $2 \%$ lignocaine indicating they are nerve-mediated. Stimulation targets were verified with hexamethonium that completely abolished pelvic nerve responses by had no effect on detrusor strips; responses to mixed bladder wall stimulation were partially reduced. The frequency-dependence of contractile activation was similar whether by pre- or postganglionic stimulation in both pigs and rats. Sildenafil reduced contractions to preganglionic stimulation significantly more than to postganglionic stimulation. Mixed pre- and postganglionic stimulation were reduced by an intermediate extent.

Conclusions: Intramural ganglia offer no frequency-dependent modulation under the experimental conditions used here and the sildenafil data are consistent with multiple sites of action underlying generation of bladder contractions. A translational aspect of these findings is discussed in terms of setting stimulation parameters for neuromodulation protocols.

Keywords: Bladder; Pelvic nerve; Nerve-mediated contractions; Sildenafil; Hexamethonium

- Research Ethics: Animal care and experimental procedures with rats were in compliance with the University of Bristol Ethics Committee and UK legislation under the Animals (Scientific Procedures) Act 1986 Amendment Regulations (SI 2012/3039) and adhered to ARRIVE guidelines.

- Conflict of Interest: No potential conflict of interest relevant to this article was reported.

\section{- HIGHLIGHTS}

- The frequency-dependence of contraction initiation in pig and rat bladder by pre- or postganglionic stimulation is similar, implying the absence of intrinsic frequency modulation by intramural ganglia. The phosphodiesterase inhibitor sildenafil reduced contractions more with pre- than with postganglionic stimulation, implying multiple sites of action.

Corresponding author: Christopher Fry (iD https://orcid.org/0000-0003-3647-5983 School of Physiology, Pharmacology and Neuroscience, University of Bristol, Bristol BS8 1TD, UK

Email: chris.fry@bristol.ac.uk

Submitted: January 9, 2021 / Accepted after revision: February 10, 2021
This is an Open Access article distributed under the terms of the Creative Commons Attribution Non-Commercial License (https://creativecommons.org/licenses/by-nc/4.0/) which permits unrestricted non-commercial use, distribution, and reproduction in any medium, provided the original work is properly cited. 


\section{INTRODUCTION}

The normal excitatory innervation of the bladder comprises preganglionic parasympathetic fibres that arise from the sacral spinal cord and project to intramural ganglia. Acetylcholine (ACh) release onto nicotinic receptors of postganglionic neurons generates action potentials that are conducted to the detrusor layer of the bladder wall (BW) and elicit contraction $[1,2]$. A question arises if the ganglion itself modulates parasympathetic signal transmission. n vagal innervation of the trachea, the ganglion is an effective filter of incoming signals [3]. In contrast, some sympathetic ganglia act as preganglionic amplifiers [4]. The situation with bladder intramural ganglia is less clear. De Groat and Saum [1] showed that low-frequency preganglionic stimulation $(<1 \mathrm{~Hz})$ recruits fewer postganglionic fibres than stimulation at higher frequencies $(5-10 \mathrm{~Hz})$, but it was not possible to show whether the frequency or intensity of preganglionic stimulation accounted for this modulation.

Determination of the physiological range of firing frequency in pre- and postganglionic nerves to the bladder is relevant, as postganglionic parasympathetic fibres release 2 transmitters: $\mathrm{ACh}$, which is released at higher frequencies $(10-40 \mathrm{~Hz})$, and adenosine triphosphate (ATP), which is released at lower frequencies $(1-10 \mathrm{~Hz})$ [5]. The normal human bladder contractile activation is solely mediated by ACh, as determined by the abolition of in vitro nerve-mediated responses with atropine [6]. This pattern is unlike that of human bladders with benign lower urinary tract pathologies, such as overactive bladder $(\mathrm{OAB})$, and the bladders of most laboratory animals, which show atropine-resistant contractions, themselves abolished by additional purinergic $\left(\mathrm{P} 2 \mathrm{X}_{1}\right)$ receptor desensitization, due to nerve-mediated ATP release. Whether ATP is a functional neurotransmitter depends on its hydrolysis rate at the nerve-muscle junction [7]. The differential control of nerve-mediated ATP and ACh release has translational potential as the selective reduction of ATP release from postganglionic fibres would attenuate a transmitter associated with $\mathrm{OAB}$ contractions in humans. It would therefore be important to measure the relationship between the frequency ranges of pre- and postganglionic stimulation that generate bladder contractions as a paradigm for artificial neuromodulation to control transmitter release selectively.

An additional aim of the study was to investigate the role of the phosphodiesterase-5 (PDE5) inhibitor sildenafil in modifying detrusor contractions. Sildenafil has a direct relaxatory action on detrusor muscle, suggesting that it may play a role in therapy for OAB. However, it also selectively reduces ATP neurotransmitter release over ACh at postganglionic parasympathetic fibres, which raises the question of whether it also influences preganglionic transmitter release. Therefore, its action on pre- and postganglionic stimulation was also tested.

These experiments used ex vivo and in vitro pig bladder preparations for 2 reasons: the physiology is similar to that of the human bladder [8], and experiments can be done, in this preliminary study, without the potential confounding effect of sympathetic hypogastric activity on ganglion function as would exist with in vivo experiments. Additional experiments with in vivo and in vitro rat preparations were done to determine whether similar data would be found in a small laboratory animal model.

\section{MATERIALS AND METHODS}

\section{Experimental Animals and Ethical Approval}

Data were collected from adult female pigs (Sus scrofa domestica, 6 months) and rats (Wistar 200-240 g). Pig bladders were obtained from the local abattoir, immediately after slaughter (Langford, Bristol, UK). Rats were sourced from Charles River UK Ltd. and housed at the University of Bristol Animal Services Unit, where they were maintained at $22^{\circ} \mathrm{C}$ in plastic cages with bedding, under a 12/12-hour light-dark environment and with free access to food and water. Animal care and experimental procedures with rats were in compliance with the University of Bristol Ethics Committee and UK legislation under the Animals (Scientific Procedures) Act 1986 Amendment Regulations (SI 2012/3039) and adhered to ARRIVE guidelines [9].

\section{Pig Preparations}

The bladder, ureters, urethra, and distal abdominal aorta with the vesical branches were removed and immediately transported to the laboratory in ice-cold, gassed Tyrode's solution. The aorta was cannulated with a $14-\mathrm{G}$ needle, the redundant branches were ligated, and the bladder was perfused $(10 \mathrm{~mL} / \mathrm{min})$ with Tyrode's solution by a peristaltic pump: drug interventions were introduced into the arterial perfusate. The preparation was put into a 20 -L closed water-jacketed $\left(36^{\circ} \mathrm{C}\right)$ chamber, into which venous outflow drained through a siphon, to maintain a humidified environment. A 6-channel double-lumen catheter was inserted into the bladder via the left ureter, secured with a Vicryl suture, and connected to a pressure transducer; the right ureter was ligated. The bladder was filled with Tyrode's solution 

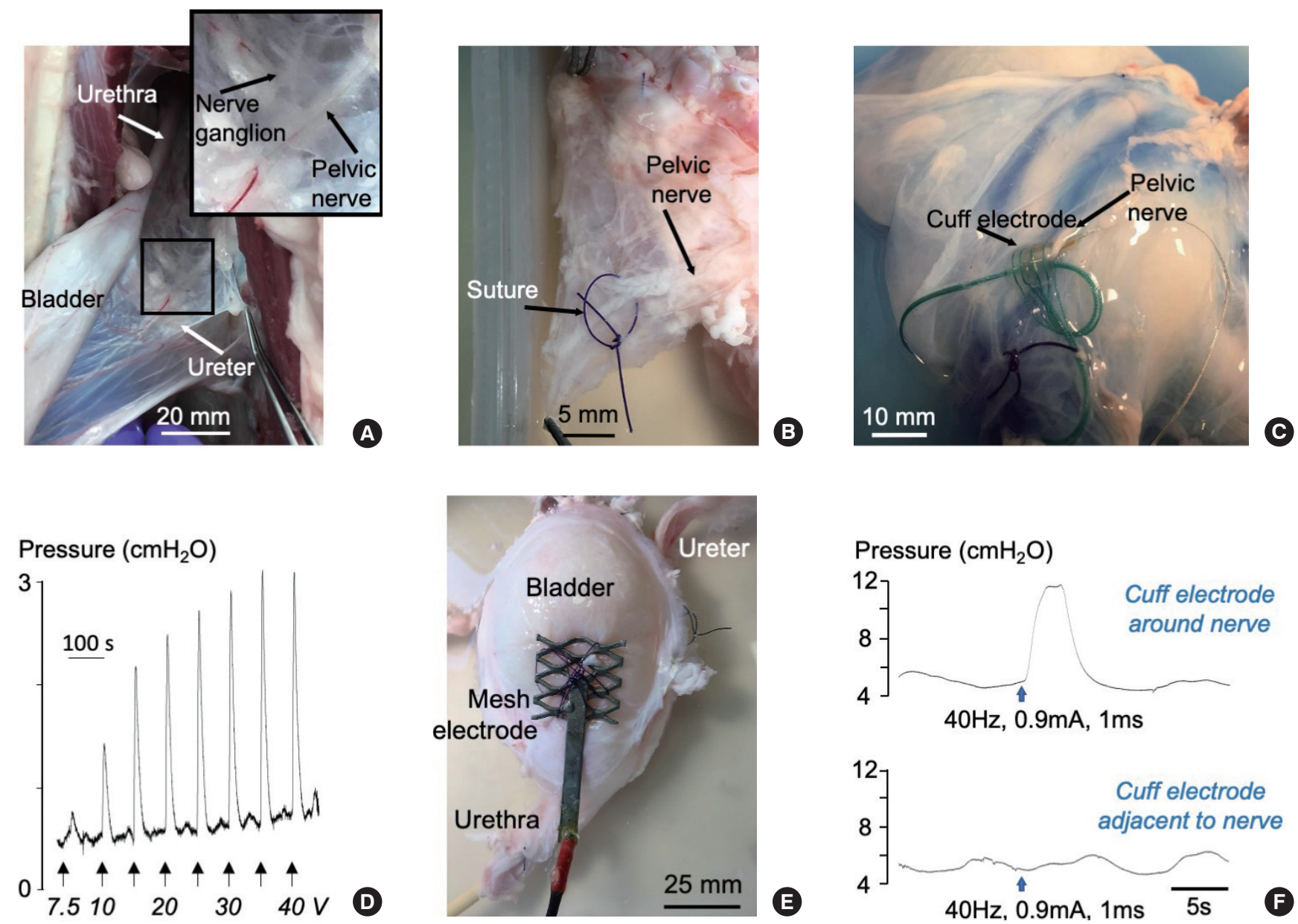

Fig. 1. Pig bladder pelvic nerve and bladder wall ex vivo preparations. (A) Dissection of the pig pelvic region to show the lower urinary tract. The inset shows the pelvic nerve and an intramural ganglion. (B) Suture around the pelvic nerve prior to placement of the cuff electrode. (C) Cuff electrode in place. (D) Intravesical pressure traces at different stimulator voltages to determine the threshold stimulation conditions. (E) Pig bladder with electrode in place for bladder wall stimulation. (F) Rat bladder, intravesical pressure traces with the cuff electrode around the pelvic nerve (upper trace) or adjacent to the nerve (lower trace).

to $150 \mathrm{~mL}$ through a 14-channel Foley catheter and drained as required. This model has been previously validated [8]. Finally, the pelvic nerves (PNs) were identified (Fig. 1A, B).

\section{Preganglionic Stimulation Ex Vivo Model}

Intravesical pressure transients were generated by PN stimulation with an isolated Grass SD9 stimulator (AstroNova, Elgin, IL, USA) via bipolar platinum-iridium cuff electrodes (miniature nerve cuff electrode, WPI, Hitchin, Herts, UK; Fig. 1C). The stimulus protocol was a 3-second train of 0.1-msec square waves $(1-40 \mathrm{~Hz})$ every 90 seconds. Before recordings, stimulation was applied to the electrode when placed about $1 \mathrm{~cm}$ from the PN to ensure absence of field stimulation to the nerve or bladder. The stimulator output voltage was varied for each bladder to obtain a threshold ( $\mathrm{V}_{\text {thr }}$ Fig. $1 \mathrm{D}, 10 \mathrm{~V}$ in this example), and recordings were obtained at twice- $\mathrm{V}_{\text {thr }}$; this was checked at intervals and did not change by more than $10 \%$ during the experiments.

\section{Pre- and Postganglionic Stimulation Ex Vivo Model}

Pressure transients were generated by direct BW stimulation with the same stimulation protocol, via custom-made titanium mesh electrodes $\left(6.25 \mathrm{~cm}^{2}\right)$ with a $0.25-\mu \mathrm{m}$ platinum-coating. An electrode was sutured to the bladder base (Fig. 1E) and a reference $\mathrm{Ag} / \mathrm{AgCl}$ electrode to the dome. 


\section{Postganglionic Stimulation In Vitro Model}

At the end of the ex vivo experiments, a BW section was cut from the mid-dome, the mucosa was removed, and a detrusor strip (5-mm length; <1-mm diameter) was prepared to record isometric contractions by electrical field stimulation (EFS), using the same protocol [5]. The pressure and tension transducers were connected to a data acquisition system (PowerLab 16/35, WPI, Hitchin, Herts, UK) running LabChart v8 software.

\section{In Vivo and In Vitro Rat Preparations}

Female rats were anaesthetised (urethane, $1.4 \mathrm{~g} / \mathrm{kg}$, intraperitoneal), the right femoral artery was cannulated to record blood pressure and heart rate, and the right femoral vein was used for fluid infusion; the trachea was cannulated to ensure airway patency. The rectal temperature was $37^{\circ} \mathrm{C}$ with a homeothermic blanket system (Harvard Apparatus, Holliston, MA, USA). Anaesthetic depth was assessed by monitoring paw withdrawal reflexes, blood pressure, and heart rate: if required, additional anaesthesia and fluids were administered. Through a midline laparotomy, the bladder was reflected to 1 side, the PN was identified and separated from the uterine wall, and a custom-made cuff electrode [10] was placed around the distal part. Intravesical pressure was monitored by a suprapubic $25-\mathrm{G}$ needle connected to a pressure transducer, after filling to $1.5 \mathrm{~mL}$. For preganglionic nerve stimulation, the same protocol was used (STMISOLA, Biopac Systems Inc., Santa Barbara, CA, USA), with threshold measurement and the lack of field stimulation (Fig. $1 F)$ checked as above. At the end of the experiment the rat was euthanised with sodium pentobarbital (200 mg/kg, intraperitoneal) and the bladder was immediately removed. A detrusor strip, after removal of the mucosa, was prepared in the same manner as for the pig preparations to record isometric contractions with postganglionic nerve stimulation.

\section{Solutions and Drugs}

Tyrode's solution contained (mM): $\mathrm{NaCl}, 118.0 ; \mathrm{NaHCO}_{3}, 24.0$; $\mathrm{NaH}_{2} \mathrm{PO}_{4}, 1.8 ; \mathrm{KCl}, 4.0 ; \mathrm{MgCl}_{2}, 1.0 ; \mathrm{CaCl}_{2}, 1.8$; sodium pyruvate, 5.0; glucose 6.0, $5 \% \mathrm{CO}_{2}-95 \% \mathrm{O}_{2}, \mathrm{pH} 7.4$. Lidocaine- $\mathrm{HCl}$ was diluted in Tyrode's solution to a final $2 \%(\mathrm{w} / \mathrm{v})$ solution [11]; hexamethonium was dissolved in Tyrode's solution to a final concentration of $300 \mu \mathrm{M}$ [12]. Sildenafil was dissolved in dimethylsulphoxide (DMSO) as a $10 \mathrm{mM}$ stock solution and diluted to $20 \mu \mathrm{M}$ in Tyrode's solution. The chemicals and drugs were obtained from Sigma-Aldrich (Gillingham, Dorset, UK).

\section{Data and Statistical Analysis}

Data were recorded with a PowerLab 16/35 acquisition system and LabChart v8 software. Because pressure transients were superimposed on spontaneous contractions, the amplitude was measured with respect to the lowest pressure immediately after relaxation (Fig. 2A, B). The pressure $(P)$ or tension (T)-frequency relations were parameterised as:

$$
\left.P(T)=\frac{P(T)_{\max } f^{m}}{f_{1 / 2}^{m}+f^{m}} \quad 1\right)
$$

where $P_{\max }\left(T_{\max }\right)$ is the maximum estimated value at high frequencies, $f$, and $f_{1 / 2}$ is the frequency required to attain $P_{\max } / 2$ $\left(T_{\max } / 2\right) ; m$ is a constant. $P-f(T-f)$ curves were also normalised to values for each curve at $40-\mathrm{Hz}$ stimulation. Data sets are given as mean \pm standard error of the mean (SEM), with $n$ equalling the number of bladders; tension values were normalised to the mean preparation cross-sectional area. Differences between data sets were tested with analysis of variance and the post hoc parametric t-test adjusted for multiple comparisons.

\section{RESULTS}

\section{Pig Bladder-Contractions to Electrical Stimulation}

Pressure or tension transients in response to electrical stimulation of the PN (Fig. 2A), BW (Fig. 2B) and isolated detrusor strips (Fig. $2 \mathrm{C}$ ) are shown. Mean ( \pm SEM) frequency-response curves are shown to the right of the sample traces. Lidocaine completely abolished responses in all 3 preparations, indicating they were all nerve-mediated. The insets show force-frequency plots for the respective modes of stimulation, and the data were fitted by equation 1 . In each preparation, 2 curves at 30 minutes apart were generated as time-controls. The respective $P_{\max }$ values for $\mathrm{PN}$ stimulation were $5.8 \pm 2.3$ and $6.2 \pm 1.8 \mathrm{~cm} \mathrm{H}_{2} \mathrm{O}(\mathrm{n}=7$, $\mathrm{P}>0.05)$ and for BW stimulation were $6.2 \pm 1.8$ and $6.7 \pm 1.1 \mathrm{~cm}$ $\mathrm{H}_{2} \mathrm{O}(\mathrm{n}=8, \mathrm{P}>0.05) . T_{\max }$ values for EFS of isolated preparations were $2.0 \pm 0.7$ and $2.1 \pm 0.3 \mathrm{mN} / \mathrm{mg}(\mathrm{n}=6, \mathrm{P}>0.05)$.

A nicotinic receptor antagonist, hexamethonium $(300 \mu \mathrm{M})$, was used to confirm the target of stimulation in the 3 preparations. Contractions were completely abolished with PN stimulation $(0 \% \pm 0 \%$ control, $\mathrm{n}=7)$, but unaffected by EFS to isolated preparations $(102.8 \% \pm 2.7 \%$ control, $\mathrm{n}=6)$ : $\mathrm{BW}$ stimulation contractions were partially reduced $(32.6 \% \pm 2.7 \%$ control, $\mathrm{n}=8$ ) but the $f_{1 / 2}$ values were not significantly different. Thus, PN stimulation was preganglionic, EFS was postganglionic, and BW stimulation elicited pre- and postganglionic responses. 

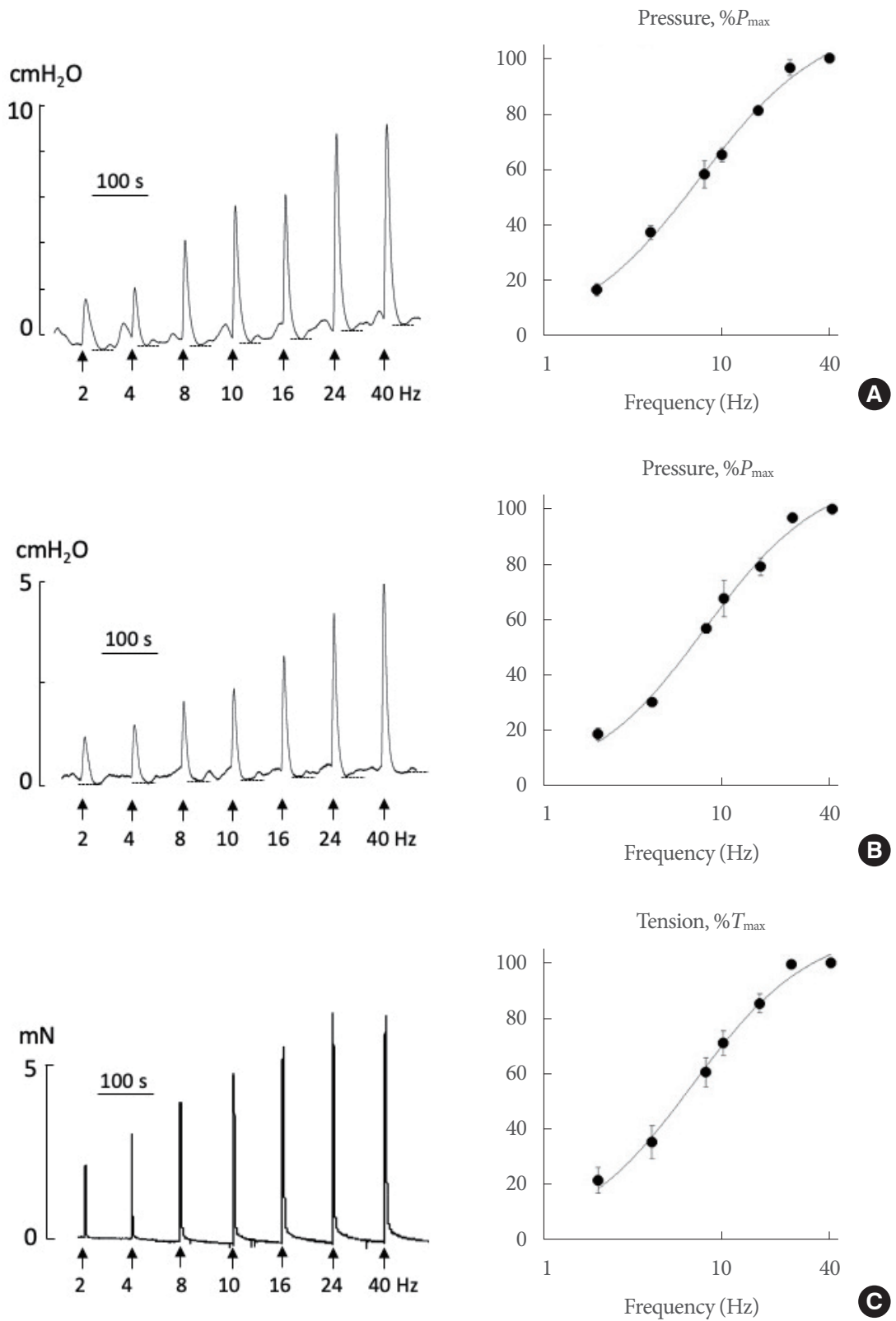

Fig. 2. Pig bladder-contractile responses to electrical stimulation. (A) Left: intravesical pressure changes in response to pelvic nerve stimulation at the frequencies indicated. Dotted lines after each contraction are the base-line from which contraction amplitude was measured. Right: mean ( \pm standard error of the mean $[\mathrm{SEM}], \mathrm{n}=7)$ data as a percentage of maximum pressure $\left(P_{\max }\right)$ at high frequencies. (B) Left: intravesical pressure changes in response to bladder wall stimulation. Right: mean $( \pm$ SEM, n $=8)$ data as a percentage of maximum pressure $\left(P_{\max }\right)$ at high frequencies. $(\mathrm{C})$ Left: isometric tension changes in a detrusor strip in response to electrical field stimulation. Right: mean data $(\mathrm{n}=6)$ as a percentage of $T_{\max }$ at high frequencies. Curves fitted to equation 1, Methods.

To test the main question of the study, the frequency-dependence of responses to the 3 modes of stimulation was calculated, as $f_{1 / 2}$ values. There was no significant difference in the $f_{1 / 2}$ values $\left(f_{1 / 2}\right.$ : the frequency for half-maximal response, equation 1) among the 3 modes of stimulation (Fig. 3A), suggesting that there was no frequency-dependent filtering by the intermediate ganglion. This result was consistent across the first and second sets of force-frequency curves from the time-control studies. In addition, the $f_{1 / 2}$ values of BW stimulation responses in the presence of hexamethonium (postganglionic responses) were 
$f_{1 / 2}, \mathrm{~Hz}$

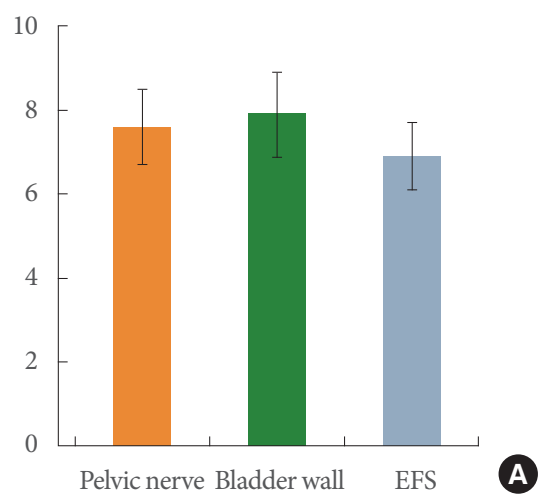

$P_{\max } / T_{\max }, \%$ control

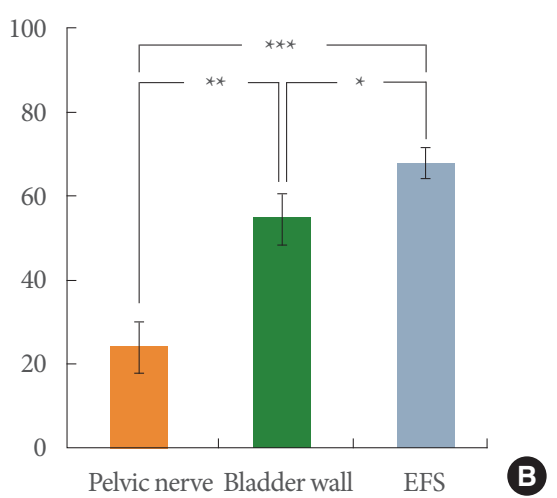

Fig. 3. Pig bladder - responses to electrical stimulation. (A) $f_{1 / 2}$ values (frequency required for half-maximum stimulation) from pelvic nerve $(n=7)$ or bladder wall $(n=8)$ stimulation of perfused bladders and from detrusor strips under electrical field stimulation (EFS, $\mathrm{n}=6)$. Data are shown as mean \pm standard error of the mean (SEM). (B) The effect of sildenafil $(20 \mu \mathrm{M})$ on maximum pressure $\left(P_{\text {max }}\right)$ or tension $\left(T_{\max }\right)$ in the pelvic nerve $(n=6)$, bladder wall $(n=6)$ or electrical field stimulation $(n=6)$. Data are shown as mean \pm SEM: ${ }^{\star} \mathrm{P}<0.05 .{ }^{* *} \mathrm{P}<0.01 .{ }^{* *} \mathrm{P}<0.001$.
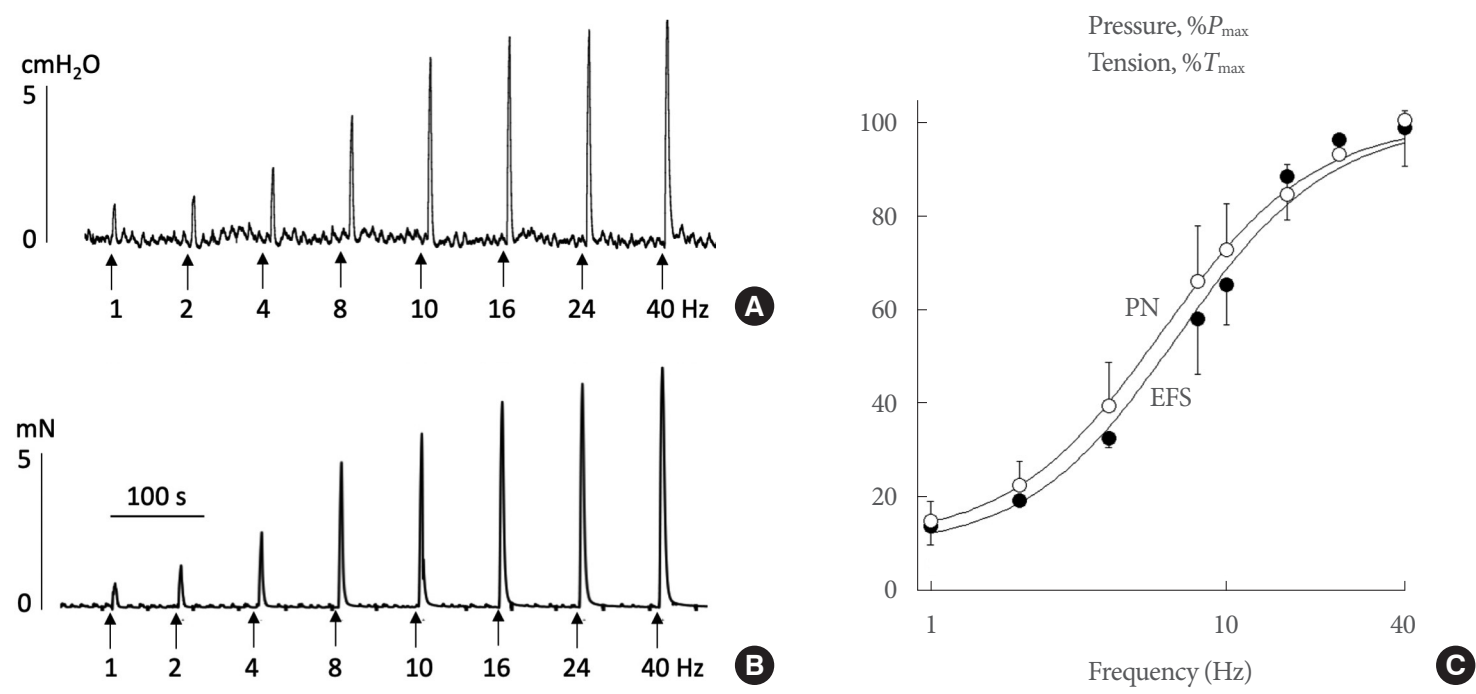

Fig. 4. Rat bladder-responses to electrical stimulation. (A) Intravesical pressure changes to pelvic nerve (PN) stimulation in control conditions. (B) Isometric tension changes from a detrusor strip after electrical field stimulation in control conditions. (C) Frequencyresponse curves to PN stimulation and electrical field stimulation, both in control conditions. Curves fitted to equation 1, Methods. Data are shown as mean \pm standard error of the mean $(n=6)$.

not significantly different from those obtained from detrusor strips (7.8 $\pm 1.7 \mathrm{~Hz}$ vs. $6.9 \pm 0.8 \mathrm{~Hz}$, respectively, $\mathrm{n}=6,6$; $\mathrm{P}>0.05)$.

\section{Pig Bladder-Responses to Sildenafil}

A second objective of the study was to identify whether sildenafil had any effect on frequency-dependent contractile responses. Force-frequency curves were generated in the presence of $20 \mu \mathrm{M}$ sildenafil, and $P_{\max }$ or $T_{\max }$ was calculated. With post- ganglionic (EFS) stimulation, sildenafil reduced $T_{\max }$ by about $30 \%$ (Fig. 3B). However, the effect on preganglionic (PN) stimulation was much greater, with $P_{\max }$ reduced by about $75 \%$. The effect on mixed pre- and postganglionic stimulation (BW) was intermediate, with a $P_{\max }$ reduction of about $45 \%$. Control experiments with DMSO, used to dissolve sildenafil for stock solutions, had no effect on isolated detrusor preparations $\left(T_{\max }\right.$ : $106.0 \% \pm 3.7 \%$ control, $\mathrm{n}=6$ ) or in $\mathrm{PN}$-stimulated preparations 
$\left(P_{\max }: 101.4 \% \pm 4.3 \%\right.$ control, $\left.\mathrm{n}=3\right)$.

\section{Rat Bladder-Contractions in Response to Electrical Stimulation}

Similar experiments were performed with PN stimulation of in vivo preparations (Fig. $4 \mathrm{~A}$ ) and $\mathrm{EFS}$ using in vitro (Fig. 4B) rat preparations. The control quantitative data for the 2 preparations were: $P_{\max } 14.3 \pm 3.1 \mathrm{~cm} \mathrm{H}_{2} \mathrm{O}(\mathrm{n}=6)$ and $\mathrm{T}_{\max } 1.8 \pm 0.4 \mathrm{mN} / \mathrm{mg}$ $(n=6)$. The respective $f_{1 / 2}$ values were: $7.3 \pm 1.4 \mathrm{~Hz}(n=6)$ and $5.4 \pm 1.7 \mathrm{~Hz}(\mathrm{n}=6)$, which were not significantly different from each other $(\mathrm{P}>0.05)$; the frequency-response curves are shown in Fig. 4C.

\section{DISCUSSION}

\section{Frequency-Dependence of Nerve-Mediated Contractions}

The main result of the study is that the frequency-dependence of contractions elicited by PN stimulation, direct stimulation of nerves in the whole BW, or in isolated detrusor preparations of the pig bladder was similar. Thus, preganglionic or postganglionic nerve stimulation generates the same frequency-dependent contractile response. There was a similar conclusion in rat preparations when in vivo and in vitro responses were compared. Moreover, the range of frequencies was similar in both species and to those recorded in other animals, as well as in human preparations $[7,13,14]$. All responses were nerve-mediated, inferred from their abolition at all frequencies by lidocaine; similar to that achieved by the neurotoxin tetrodotoxin [13]. This implies that action potential transmission between preand postganglionic fibres through the BW intramural ganglia is not frequency-modulated under these experimental conditions. Hypogastric sympathetic fibres do project to intramural ganglia, which could exert modulatory influences on intramural transmission, as exemplified in cat and dog preparations [13, 15]; however, this was not explored in this study. It was also of interest that pure postganglionic nerve stimulation in pig preparations, whether by EFS of isolated preparations or by BW stimulation in the presence of hexamethonium, produced the same frequency-dependent responses. This implies that dissection of muscle strips itself does not alter the characteristics of nerve-mediated responses.

\section{Effects of Interventions}

The nicotine receptor antagonist, hexamethonium, completely abolished PN-mediated contractions in the ex vivo perfused pig bladder, which is interpreted as indicating that ACh is the sole transmitter acting on ganglionic nicotinic receptors. Similar contractions induced in anaesthetised rats were only partially abolished (not shown), and higher doses could not be used as the physiological status of the preparation would have been compromised. This limitation highlights an advantage of the $e x$ vivo arterially-perfused bladder preparation, when higher doses of agents might be required. The partial abolition by hexamethonium of responses to direct pig BW stimulation implies that both pre- and postganglionic fibres are stimulated, corroborated by a lack of action on EFS responses in detrusor strips. Of interest, functional nicotinic receptors have been demonstrated in bladder urothelial cells and their modulation may contribute to the nicotine-induced increase of the cystometric intercontraction interval [16]. However, the experiments here using detrusor strips, denuded of mucosa, were not consistent with the presence of functional nicotinic receptors in the smooth muscle layer.

Sildenafil, a PDE5 inhibitor, increases intracellular cGMP and is associated with a relaxatory effect on isolated detrusor preparations. This may be due to a direct effect on detrusor contractile function [17], as well as inhibition of nerve-mediated release from postganglionic fibres, although ACh release remains intact [5]. The results presented here imply that sildenafil has actions at sites other than the BW, as the proportional reduction of contractile responses was significantly greater with preganglionic stimulation than with postganglionic stimulation. An interpretation is that sildenafil also affects preganglionic transmission. Several studies have demonstrated both stimulatory or inhibitory actions of the NO-cGMP pathway on transmitter release in central and peripheral pathways $[18,19]$, implying there are multiple pathways that regulate release [20]. However, it is evident that agents such as PDE5 inhibitors that may be used as therapeutic agents on peripheral targets also have central actions; with respect to the lower urinary tract, this would further depress bladder function.

\section{Translational Potential for Neuromodulation}

These data are useful to identify a frequency range for bladder stimulation using neuromodulation devices, where too low a frequency would achieve inadequate stimulation and too high a frequency would reduce stimulator efficiency and potentially tissue damage. Sacral nerve stimulators have pulse rates of about 1-150 Hz, with individual pulses of 50-500 $\mu \mathrm{sec}$ [21], which may be compared to the $100-\mu$ sec pulses at $1-40 \mathrm{~Hz}$ in 
the current study. A short pulse width $(<50-100 \mu \mathrm{sec})$ is important, as this can selectively stimulate nerve fibres without direct action on smooth muscle, due to the different membrane time constants of these 2 cell types. Recent studies of rechargeable sacral nerve stimulators have shown success and patient satisfaction with devices delivering $200-\mu$ s pulses at about 14 $\mathrm{Hz}$ [22]. More recently, intravesical devices are also being trialled and a recent study [23] to manage OAB contractions used 200- $\mu$ sec pulses at frequencies between 5 and $50 \mathrm{~Hz}$. The use of isolated detrusor preparations is an efficient way to characterise the properties of nerve-mediated contractions. The study shows that the frequency-dependent properties of isolated preparations can be used to set appropriate neurostimulation variables applied to the PN or BW.

\section{AUTHOR CONTRIBUTION STATEMENT}

- Conceptualization: $B V, C F$

- Data curation: $D K, J C, B C, T L$

- Formal analysis: $D K, J C, B C, T L, C F$

- Funding acquisition: $A K, C F$

- Methodology: $B V, C F$

- Project administration: $C F$

- Visualization: $C F$

-Writing-original draft: $C F$

- Writing-review \& editing: $A K, B V, T L, C F$

\section{ORCID}

$\begin{array}{ll}\text { Darryl Kitney } & 0000-0003-4636-3870 \\ \text { John Crook } & 0000-0002-1181-593 \mathrm{X} \\ \text { Basu Chakrabarty } & 0000-0002-7320-4931 \\ \text { Anthony Kanai } & 0000-0003-4780-7592 \\ \text { Bahareh Vahabi } & 0000-0002-7186-0943 \\ \text { Christopher Fry } & 0000-0003-3647-5983\end{array}$

\section{REFERENCES}

1. de Groat WC, Saum WR. Synaptic transmission in parasympathetic ganglia in the urinary bladder of the cat. J Physiol 1976;256:13758.

2. de Groat WC, Nadelhaft I, Milne RJ, Booth AM, Morgan C, Thor K. Organization of the sacral parasympathetic reflex pathways to the urinary bladder and large intestine. J Auton Nerv Syst 1981;3:13560.
3. Moffatt JD, Dumsday B, McLean JR. Characterization of non-adrenergic, non-cholinergic inhibitory responses of the isolated guinea-pig trachea: differences between pre- and post-ganglionic nerve stimulation. Br J Pharmacol 1999;128:459-64.

4. Rimmer K, Horn JP. Weak and straddling secondary nicotinic synapses can drive firing in rat sympathetic neurons and thereby contribute to ganglionic amplification. Front Neurosci 2010;1:130.

5. Chakrabarty B, Ito H, Ximenes M, Nishikawa N, Vahabi B, Kanai $\mathrm{AJ}$, et al. Influence of sildenafil on the purinergic components of nerve-mediated and urothelial ATP release from the bladder of normal and spinal cord injured mice. Br J Pharmacol 2019;176: 2227-37.

6. Bayliss M, Wu C, Newgreen D, Mundy AR, Fry CH. A quantitative study of atropine-resistant contractile responses in human detrusor smooth muscle, from stable, unstable and obstructed bladders. J Urol 1999;162:1840-7.

7. McCarthy CJ, Ikeda I, Skennerton D, Chakrabarty B, Kanai AJ, Jabr RI, et al. Characterisation of nerve-mediated ATP release from bladder detrusor muscle and its pathological implications. Br J Pharmacol 2019;176:4720-30.

8. Parsons BA, Drake MJ, Gammie A, Fry CH, Vahabi B. The validation of a functional, isolated pig bladder model for physiological experimentation. Front Pharmacol 2012;3:52.

9. Percie du Sert N, Hurst V, Ahluwalia A, Alam S, Avey MT, Baker M, et al. The ARRIVE guidelines 2.0: updated guidelines for reporting animal research. J Physiol 2020;598:3793-801.

10. Crook JJ, Brouillard CB, Irazoqui PP, Lovick TA. Chronic implantation of cuff electrodes on the pelvic nerve in rats is well tolerated and does not compromise afferent or efferent fibre functionality. J Neural Eng 2018;15:024001.

11. Nakamura T, Popitz-Bergez F, Birknes J, Strichartz GR. The critical role of concentration for lidocaine block of peripheral nerve in vivo: studies of function and drug uptake in the rat. Anesthesiology 2003;99:1189-97.

12. Yu Y, Daugherty S, de Groat WC. Effects of nicotinic receptor agonists on bladder afferent nerve activity in an in vitro bladder-pelvic nerve preparation. Brain Res 2016;1637:91-101.

13. de Groat WC, Saum WR. Sympathetic inhibition of the urinary bladder and of pelvic ganglionic transmission in the cat. J Physiol 1972:220:297-314.

14. Fry CH, Bayliss M, Young JS, Hussain M. Influence of age and bladder dysfunction on the contractile properties of isolated human detrusor smooth muscle. BJU Int 2011;108:E91-6.

15. Barbe MF, Gomez-Amaya SM, Salvadeo DM, Lamarre NS, Tiwari E, Cook S, et al. Clarification of the innervation of the bladder, ex- 
ternal urethral sphincter and clitoris: a neuronal tracing study in female mongrel hound dogs. Anat Rec 2018;301:1426-41.

16. Beckel JM, Kanai AJ, Lee SJ, de Groat WC, Birder LA. Expression of functional nicotinic acetylcholine receptors in rat urinary bladder epithelial cells. Am J Physiol Renal Physiol 2006;290:F103-10.

17. Oger S, Behr-Roussel D, Gorny D, Lebret T, Validire P, Cathelineau $\mathrm{X}$, et al. Signalling pathways involved in sildenafil-induced relaxation of human bladder dome smooth muscle. Br J Pharmacol 2010;160:1135-43.

18. Herring N, Paterson DJ. Nitric oxide-cGMP pathway facilitates acetylcholine release and bradycardia during vagal nerve stimulation in the guinea-pig in vitro. J Physiol 2004;535;507-18.

19. Kilbinger H. Modulation of acetylcholine release by nitric oxide. Prog Brain Res 1996;109;219-24.
20. Wang X, Robinson PJ. Cyclic GMP-dependent protein kinase and cellular signaling in the nervous system. J Neurochem 2002;68;44356.

21. Coolen RL, Groen J, Blok BF. Electrical stimulation in the treatment of bladder dysfunction: technology update. Med Devices 2019;12:337-45.

22. Blok BF, van Kerrebroeck P, de Wachter S, Ruffion A, van der Aa F, Jairam R, et al. Programming settings and recharge interval in a prospective study of a rechargeable sacral neuromodulation system for the treatment of overactive bladder. Neurourol Urodyn 2018; 37:S17-22.

23. Yune JJ, Shen JK, Pierce MA, Hardesty JS, Kim J, Siddighi S. Intravesical electrical stimulation treatment for overactive bladder: an observational study. Invest Clin Urol 2018;59:246-51. 